\title{
Short and Efficient Certificate-Based Signature ${ }^{\star}$
}

\author{
Joseph K. Liu, Feng Bao, and Jianying Zhou \\ Cryptography and Security Department, \\ Institute for Infocomm Research, Singapore \\ \{ksliu, baofeng, jyzhou\}@i2r.a-star.edu.sg
}

\begin{abstract}
In this paper, we propose a short and efficient certificatebased signature (CBS) scheme. Certificate-based cryptography proposed by Gentry [6] combines the merit of traditional public key cryptography (PKI) and identity based cryptography, without use of the costly certificate chain verification process and the removal of key escrow security concern. Under this paradigm, we propose the shortest certificate-based signature scheme in the literature. We require one group element for the signature size and public key respectively. Thus the public information for each user is reduced to just one group element. It is even shorter than the state-of-the-art PKI based signature scheme, which requires one group element for the public key while another group element for the certificate. Our scheme is also very efficient. It just requires one scalar elliptic curve multiplication for the signing stage. Our CBS is particularly useful in power and bandwidth limited environment such as Wireless Cooperative Networks.
\end{abstract}

\section{Introduction}

Different Cryptosystems: Public Key Infrastructure (PKI). In a traditional public key cryptography (PKC), a user Alice signs a message using her private key. A verifier Bob verifies the signature using Alice's public key. However, the public key is just merely a random string and it does not provide authentication of the signer by itself. This problem can be solved by incorporating a certificate generated by a trusted party called the Certificate Authority (CA). The hierarchical framework is called the public key infrastructure (PKI). Prior to the verification of a signature, Bob needs to obtain Alice's certificate in advance and verify the validity of her certificate. If it is valid, Bob extracts the corresponding public key which is then used to verify the signature. This approach seems inefficient, in particular when the number of users is large.

IDENTITY-BASED CRYPTOGRAPHY (IBC). Identity-based cryptography (IBC) solves the aforementioned problem by using Alice's identity (or email address) which is an arbitrary string as her public key while the corresponding private key is a result of some mathematical operation that takes as input the user's identity and the master secret key of a trusted authority, referred to as "Private Key Generator (PKG)". The main disadvantage of identity-based cryptography

\footnotetext{
* The work is supported by A*STAR project SEDS-0721330047.
} 
is an unconditional trust to the PKG. Hence, IBC is only suitable for a closed organization where the PKG is completely trusted by everyone in the group.

Certificate-Based CRyptography (CBC). To integrate the merits of IBC into PKI, Gentry [6] introduced the concept of certificate-based encryption (CBE). A CBE scheme combines a public key encryption scheme and an identity based encryption (IBE) scheme between a certifier and a user. Each user generates his/her own private and public keys and requests a certificate from the CA while the CA uses the key generation algorithm of an IBE 4 s scheme to generate the certificate from the user identity and his public key. The certificate is implicitly used as part of the user decryption key, which is composed of the usergenerated private key and the certificate. Although the CA knows the certificate, it does not have the user private key. Thus it cannot decrypt any ciphertexts. In addition to CBE, the notion of certificate-based signature (CBS) was first suggested by Kang et al. [9]. In a CBS scheme, the signing process requires both the user private key and his certificate, while verification only requires the user public key.

Certificateless CRYPtography (CLC). Certificateless cryptography [1] is another stream of research, which is to solve the key escrow problem inherited by IBC. In a CLC, the CA generates a partial secret key from the user's identity using the master secret key, while the user generates his/her own private and public keys which are independent to the partial secret key. Decryption and signature generation require both the user private key and partial secret key. The concept is similar to CBC. The main different is the generation of the partial secret key in CLC (it is called certificate in CBC). Here the CA does not require the user's public key for the generation of the partial secret key, while in CBC the CA does require the user's public key for the generation of the certificate.

Level of Trust: Girault 7] defined three levels of trust of a PKG or CA: Level 1: The PKG can compute users' secret keys and, therefore, can impersonate any user without being detected. ID-based signatures belong to this level. Level 2: The PKG cannot compute users' secret keys. However, the PKG can still impersonate any user without being detected. Certificateless signatures belong to this level.

Level 3: The PKG cannot compute users' secret keys, and the PKG cannot impersonate any user without being detected. This is the highest level of trust. Traditional PKI signatures and certificate-based signatures belong to this level.

We summarize different kinds of cryptosystems in table 1 . We compare the public information (per user) and the level of trust between different cryptosystems. "ID" represents the identity of the user. "PK" represents the public key of the user. "Cert" represents the certificate. Note that although certificate-based cryptosystem also requires a certificate, that one is not public information. In contrast, it is part of secret information and should be kept secret by the user. 
Table 1. Comparison of different cryptosystems

\begin{tabular}{|l||c|c|}
\hline Cryptosystem & Public Information & Level of Trust \\
\hline \hline Traditional PKI & ID, PK, Cert & 3 \\
\hline ID-based & ID & 1 \\
\hline Certificateless & ID, PK & 2 \\
\hline Certificate-Based & ID, PK & 3 \\
\hline
\end{tabular}

Application of CBS: CBS can be very useful in many scenarios. It can provide the highest level of trust, while also maintaining the shortest length and most efficient verification. It is especially useful in those environments such that computation power is very limited, or communication bandwidth is very expensive. Wireless Cooperative Networks (WCN) including wireless sensor networks or mesh networks are good examples of such environments. The efficient verification can let the node to enjoy a reduced complexity or energy consumption. On the other side, the elimination of certificate in the verification provides a shorter length of total transmitted data. In the case of WCNs, communication bandwidth is a very expensive resource. A shorter length means a cheaper cost to provide the same level of service without compromising security.

Contribution: We propose the shortest certificate-based signature (CBS). The signature size and public key of our scheme are both of one element respectively, which is around 160 bits. All previous CBS schemes 910311 require at least two elements for the signature size, except for the construction by aggregated BLS signature scheme [5]. Our scheme is comparable to the shortest PKI-based signature [5] which also contains one element for both the public key and signature size. However, as a PKI-based signature, a certificate should be attached to the public key. A certificate itself is a signature (signed by the CA). Thus the total length of the public information (public key + certificate) should be at least two elements. Our scheme only requires one element for both the signature and public information while maintaining the same level of trust as other traditional PKI-based signatures. In addition, our scheme requires two pairings for the verification, while the CBS from aggregated BLS requires three pairings. We also note that the CBS from aggregated BLS signature has not been formally proven secure, though we still include it in our efficiency comparison.

Our scheme can be proven secure in the random oracle model (ROM), using the weak modified $k$-CAA assumption. The contribution of our scheme is summarized in table 2, by comparing with other most efficient state-of-the-art schemes of different cryptosystems.

\footnotetext{
${ }^{1}$ It is easy to observe that by using an aggregated BLS signature, one can get a CBS. The CA generates the certificate as a BLS signature. The user signs the message and his public key using his own secret key to generate another BLS signature. These two signatures are aggregated together to form the final signature. In this way, the length of the final signature is still one element.
} 
Table 2. Comparison of different signature schemes with different cryptosystems

\begin{tabular}{|c||c|c|c|c|c|c|}
\hline Cryptosystem & $\begin{array}{c}\text { State-of } \\
\text {-the-art } \\
\text { scheme }\end{array}$ & $\begin{array}{c}\text { Number } \\
\text { of pairing } \\
\text { (veri.) }\end{array}$ & $\begin{array}{c}\text { Security } \\
\text { Model }\end{array}$ & $\begin{array}{c}\text { Length of } \\
\text { Public Info. } \\
\text { (per user) }\end{array}$ & $\begin{array}{c}\text { Length } \\
\text { of } \\
\text { Sign. }\end{array}$ & $\begin{array}{c}\text { Level } \\
\text { of } \\
\text { Trust }\end{array}$ \\
\hline \hline PKI & {$[5]$} & 4 & ROM & 320 bits + ID & 160 bits & 3 \\
\hline ID-based & {$[8]$} & 2 & ROM & ID & 320 bits & 1 \\
\hline Certificateless & {$[14$} & 2 & ROM & 320 bits + ID & 160 bits & 2 \\
\hline $\begin{array}{c}\text { Certificate } \\
\text { Based }\end{array}$ & $\begin{array}{c}\text { Agg. } \\
\text { BLS }\end{array}$ & 3 & $\begin{array}{c}\text { not formally } \\
\text { proven }\end{array}$ & 160 bits + ID & 160 bits & 3 \\
\hline $\begin{array}{c}\text { Certificate } \\
\text { Based }\end{array}$ & $\begin{array}{c}\text { our prop. } \\
\text { scheme }\end{array}$ & 2 & ROM & 160 bits + ID & 160 bits & 3 \\
\hline
\end{tabular}

\section{Preliminaries and Security Model}

\subsection{Mathematical Assumptions}

Definition 1 ( $k$-Collusion Attack Algorithm Assumption ( $k$-CAA)). [12] The $k$-CAA problem in $\mathbb{G}$ is defined as follow: For some $x, h_{1}, \cdots, h_{k} \in \mathbb{Z}_{p}^{*}$ and $g \in \mathbb{G}$, given $g, g^{x}$ and $k$ pairs $\left(h_{1}, g^{\left(x+h_{1}\right)^{-1}}\right), \ldots,\left(h_{k}, g^{\left(x+h_{k}\right)^{-1}}\right)$, output a new pair $\left(h^{*}, g^{\left(x+h^{*}\right)^{-1}}\right)$ for some $h^{*} \notin\left\{h_{1}, \ldots, h_{k}\right\}$. We say that the $(t, \epsilon) k-C A A$ assumption holds in $\mathbb{G}$ if no t-time algorithm has advantage at least $\epsilon$ in solving the $k$-CAA problem in $\mathbb{G}$.

Definition 2 (Weak Modified $k$-CAA Assumption2). The Weak Modified $k$-CAA problem in $\mathbb{G}$ is defined as follow: For some $x, a, b, h_{1}, \cdots, h_{k} \in \mathbb{Z}_{p}^{*}$ and $g \in \mathbb{G}$, given $g, g^{x}, g^{a}, g^{b}$ and $k$ pairs $\left(h_{1},\left(g^{a b}\right)^{\left(x+h_{1}\right)^{-1}}\right), \ldots,\left(h_{k},\left(g^{a b}\right)^{\left(x+h_{k}\right)^{-1}}\right)$, output either a new pair $\left(h^{*},\left(g^{a b}\right)^{\left(x+h^{*}\right)^{-1}}\right)$ for some $h^{*} \notin\left\{h_{1}, \ldots, h_{k}\right\}$, or $g^{a b}$. We say that the $(t, \epsilon)$ Weak Modified $k$-CAA assumption holds in $\mathbb{G}$ if no $t$-time algorithm has advantage at least $\epsilon$ in solving the Weak Modified $k$-CAA problem in $\mathbb{G}$.

\subsection{Security Model}

Definition 3. A certificate-based signature (CBS) scheme is defined by six algorithms:

- Setup is a probabilistic algorithm taking as input a security parameter. It returns the certifier's master key msk and public parameters param. Usually this algorithm is run by the $C A$.

- UserKeyGen is a probabilistic algorithm that takes param as input. When run by a client, it returns a public key PK and a secret key usk.

${ }^{2}$ We define a weaker version of the Modified $k$-CAA Assumption [14. The only difference in this version is that, we do not require $g^{b x}$ as input. It is defined as follow: 
- Certify is a probabilistic algorithm that takes as input (msk, $\tau$, param, PK, ID) where ID is a binary string representing the user information. It returns Cert' ${ }_{\tau}^{\prime}$ which is sent to the client. Here $\tau$ is a string identifying a time period.

- Consolidate is a deterministic certificate consolidation algorithm taking as

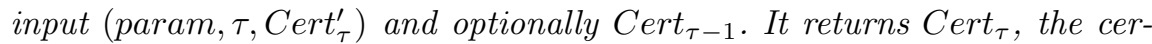
tificate used by a client in time period $\tau$.

- Sign is a probabilistic algorithm taking as input ( $\tau$, param $_{1}$, Cert $_{\tau}$, usk) where $m$ is a message. It outputs a ciphertext $\sigma$.

- Verify is a deterministic algorithm taking (param, PK,ID, $\sigma$ ) as input in time period $\tau$. It returns either valid indicating a valid signature, or the special symbol $\perp$ indicating invalid.

We require that if $\sigma$ is the result of applying algorithm Sign with intput ( $\tau$, param, $m$, Cert $\left._{\tau}, u s k\right)$ and $(u s k, P K)$ is a valid key-pair, then valid is the result of applying algorithm Verify on input (param, PK,ID, $\sigma)$, where Cert w $_{\tau}$ is the output of Certify and Consolidate algorithms on input (msk, param, $\tau, P K)$. That is, we have $\operatorname{Verify}_{P K, I D}\left(\operatorname{Sign}_{\tau, C_{\text {Cert }}, \text { usk }}(m)\right)=$ valid We also note that a concrete $C B S$ scheme may not involve certificate consolidation. In this situation, algorithm Consolidate will simply output Cert $_{\tau}=$ Cert $_{\tau}^{\prime}$.

In the rest of this paper, for simplicity, we will omit Consolidate and the time identifying string $\tau$ in all notations.

The security of CBS is defined by two different games and the adversary chooses which game to play. In Game 1, the adversary models an uncertified entity while in Game 2, the adversary models the certifier in possession of the master key msk attacking a fixed entity's public key. We use the enhanced model by Li et al. [10] which captures key replacement attack in the security of Game 1.

Definition 4 (CBS Game 1 Existential Unforgeable). The challenger runs Setup, gives param to the adversary $\mathcal{A}$ and keeps msk to itself. The adversary then interleaves certification and signing queries as follows:

- On user-key-gen query $(I D)$, if ID has been already created, nothing is to be carried out. Otherwise, the challenger runs the algorithm UserKeyGen to obtains a secret/public key pair $\left(u s k_{I D}, P K_{I D}\right)$ and adds to the list L. In this case, ID is said to be 'created'. In both cases, $P K_{I D}$ is returned.

- On corruption query $(I D)$, the challenger checks the list $L$. If ID is there, it returns the corresponding secret key usk $k_{I D}$. Otherwise nothing is returned.

- On certification query $(I D)$, the challenger runs Certify on input (msk, param, $P K, I D)$, where $P K$ is the public key returned from the user-key-gen query, and returns Cert.

- On key-replace query (ID, PK, usk), the challenger checks if PK is the public key corresponding to the secret key usk. If yes, it updates the secret/public key pair to the list $L$. Otherwise it outputs $\perp$ meaning invalid operation.

- On signing query $(I D, P K, m)$, the challenger generates $\sigma$ by using algorithm Sign. 
Finally $\mathcal{A}$ outputs a signature $\sigma^{*}$, a message $m^{*}$ and a public key $P K^{*}$ with user information $I D^{*}$. The adversary wins the game if (1) $\sigma^{*}$ is a valid signature on the message $m^{*}$ under the public key $P K^{*}$ with user information $I^{*}$, where $P K^{*}$ is either the one returned from user-key-gen query or a valid input to the key-replace query. (2) ID* has never been submitted to the certification query. (3) $\left(I D^{*}, P K^{*}, m^{*}\right)$ has never been submitted to the signing query.

We define $\mathcal{A}$ 's advantage in this game to be $\operatorname{Adv}(\mathcal{A})=\operatorname{Pr}[\mathcal{A}$ wins $]$.

Definition 5 (CBS Game 2 Existential Unforgeable). The challenger runs Setup, gives param and msk to the adversary $\mathcal{A}$. The adversary interleaves user-key-gen queries, corruption queries and signing queries as in Game 1. But different from Game 1, the adversary is not allowed to replace any public key.

Finally $\mathcal{A}$ outputs a signature $\sigma^{*}$, a message $m^{*}$ and a public key $P K^{*}$ with user information ID*. The adversary wins the game if (1) $\sigma^{*}$ is a valid signature on the message $m^{*}$ under the public key $P K^{*}$ with user information ID*. (2) $P K^{*}$ is an output from user-key-gen query. (3) ID* has never been submitted to corruption query. (4) $\left(I D^{*}, P K^{*}, m^{*}\right)$ has never been submitted to the signing query.

We define $\mathcal{A}$ 's advantage in this game to be $\operatorname{Adv}(\mathcal{A})=\operatorname{Pr}[\mathcal{A}$ wins $]$.

We note that our model does not support security against Malicious Certifier. That is, we assume that the certifier generates all public parameters honest, according to the algorithm specified. The adversarial certifier is only given the master secret key, instead of allowing to generate all public parameters. Although malicious certifier has not been discussed in the literature, similar concept of Malicious Key Generation Centre (KGC) [2] has been formalized in the area of certificateless cryptography.

\section{The Proposed Scheme}

\subsection{Construction}

Setup. Select a pairing $e: \mathbb{G} \times \mathbb{G} \rightarrow \mathbb{G}_{T}$ where the order of $\mathbb{G}$ is $p$. Let $g$ be a generator of $\mathbb{G}$. Let $H_{1}:\{0,1\}^{*} \rightarrow \mathbb{G}$ and $H_{2}:\{0,1\}^{*} \rightarrow \mathbb{Z}_{p}$ be two collisionresistant cryptographic hash functions. Randomly select $\alpha \in_{R} \mathbb{Z}_{p}$ and compute $g_{1}=g^{\alpha}$. The public parameters param are $\left(e, \mathbb{G}, \mathbb{G}_{T}, p, g, g_{1}\right)$ and the master secret key $m s k$ is $\alpha$.

UserKeyGen. User selects a secret value $x \in \mathbb{Z}_{p}$ as his secret key $u s k$, and computes his public key $P K$ as $Y=g^{x}$.

Certify. To construct the certificate for user with public key $P K$ and binary string $I D$, the CA computes $C=H_{1}(I D, P K)^{\alpha}$.

Sign. To sign a message $m \in\{0,1\}^{*}$, the signer with public key $P K$ (and user information $I D)$, certificate $C$ and secret key $x$, compute $\sigma=C^{\frac{1}{x+H_{2}(m, I D, P K)}}$

Verify. Given a signature $\sigma$ for a public key $P K$ and user information $I D$ on a message $m$, a verifier checks whether $e\left(\sigma, Y \cdot g^{H_{2}(m, I D, P K)}\right) \stackrel{?}{=} e\left(H_{1}(I D, P K), g_{1}\right)$ Output valid if it is equal. Otherwise output $\perp$. 


\section{$3.2 \quad$ Analysis}

For efficiency, the computation requirement of our scheme is very light. In the signing stage, we just require one exponentiation, which is the minimum among every signature scheme (except for online/offline signature schemes [13]). For verification, we require two pairing operations, which is generally acceptable as verification is usually done in relatively powerful device such as computer or PDA. Our scheme can be implemented in many devices, such as personal computers, PDAs, mobile phones, wireless sensors or smart cards.

For security, our scheme can be proven secure by the following theorems:

Theorem 1 (Game 1). The CBS scheme proposed in this section is $(\epsilon, t)$ existential unforgeable against Game 1 adversary with advantage at most $\epsilon$ and runs in time at most $t$, assuming that the $\left(\epsilon^{\prime}, t^{\prime}\right)$-Weak Modified $k$-CAA assumption holds in $\mathbb{G}$, where $\epsilon^{\prime} \geq\left(1-\frac{1}{q_{k}}\right)^{q_{e}+q_{r}}\left(1-\frac{1}{q_{s}+1}\right)^{q_{s}} \frac{1}{\left(q_{s}+1\right) q_{k}} \epsilon, t^{\prime}=t$ where $q_{k}$, $q_{r}, q_{e}, q_{s}$ are the numbers of queries made to the User-key-gen Query, Corruption Query, Certification Query and Signing Query respectively.

Proof. Assume there exists a Game 1 adversary $\mathcal{A}$. We construct another PPT $\mathcal{B}$ that makes use of $\mathcal{A}$ to solve the Weak Modified $k$-CAA problem with probability at least $\epsilon^{\prime}$ and in time at most $t^{\prime}$. We use a similar approach as in 14.

Let $g$ be a generator of $\mathbb{G}$. Let $x, a, b \in \mathbb{Z}_{p}^{*}$ be three integers and $h_{1}, \ldots, h_{k} \in \mathbb{Z}_{p}^{*}$ be $k$ integers. $\mathcal{B}$ is given the problem instance $g, g^{x}, g^{a}, g^{b},\left(h_{1},\left(g^{a b}\right)^{\left(x+h_{1}\right)^{-1}}\right)$, $\ldots,\left(h_{k},\left(g^{a b}\right)^{\left(x+h_{k}\right)^{-1}}\right) \cdot \mathcal{B}$ is asked to output $g^{a b}$ or $\left(h^{*},\left(g^{a b}\right)^{\left(x+h^{*}\right)^{-1}}\right)$ for some $h^{*} \notin\left\{h_{1}, \ldots, h_{k}\right\}$.

Setup. $\mathcal{B}$ simulates the environment and the oracles which $\mathcal{A}$ can access. We regard the hash function $H_{1}$ and $H_{2}$ as random oracles. $\mathcal{B}$ first sets $g_{1}=g^{a}$ and gives the parameter param $=\left(e, \mathbb{G}, \mathbb{G}_{T}, p, g, g_{1}\right)$ to $\mathcal{A}$.

Oracles Simulation. $\mathcal{B}$ simulates all oracles as follow:

(User-key-gen (and $H_{1}$ random oracle) Query .) At the beginning $\mathcal{B}$ first randomly chooses a number $t \in\left\{1, \ldots, q_{k}\right\}$. $\mathcal{B}$ also maintains a list $L_{U}$ which stores the secret/public key pair of the corresponding identity. $\mathcal{A}$ can query this oracle by submitting an identity $I D_{i}$. If the public key pair is already created, $\mathcal{B}$ retrieves them from the list $L_{U}$. Otherwise,

1. If $i \neq t, \mathcal{B}$ chooses $d_{i}, x_{i} \in_{R} \mathbb{Z}_{p}^{*}$ and sets $P K_{i}=g^{x_{i}}, H_{1}\left(I D_{i}, P K_{i}\right)=g^{d_{i}}$. The corresponding certificate is $C_{I D_{i}}=H_{1}\left(I D_{i}, P K_{i}\right)^{a}=\left(g^{a}\right)^{d_{i}}$ and the user secret key is $x_{i} \cdot \mathcal{B}$ stores $\left(d_{i}, x_{i}, P K_{i}, I D_{i}\right)$ to the list $L_{U}$.

2. If $i=t, \mathcal{B}$ sets $P K_{i}=g^{x}$ and $H_{1}\left(I D_{i}, P K_{i}\right)=g^{b}$. In this case, the certificate and the user secret key are unknown to $\mathcal{B}$.

(Corruption Query.) Given an identity $I D_{i}, \mathcal{B}$ outputs $\perp$ if $I D_{i}=I D_{t}$ or $I D_{i}$ has not been created. Otherwise, $\mathcal{B}$ returns $x_{i}$ from the list $L_{U}$.

(Certification Query.) Given an identity $I D_{i}, \mathcal{B}$ outputs $\perp$ if $I D_{i}=I D_{t}$ or $I D_{i}$ has not been created. Otherwise, $\mathcal{B}$ returns $g^{a d_{i}}$ where $d_{i}$ is retrieved from the list $L_{U}$. 
(Key-replace Query.) Given an identity $I D_{i}$, a new public key pair $P K_{i}, u_{s k}, \mathcal{B}$ checks whether $P K_{i}$ is the corresponding public key of $u s k_{i}$. If yes, $\mathcal{B}$ replaces the new public key pair into the list $L_{U}$. Otherwise, outputs $\perp$.

(Signing (and $\mathrm{H}_{2}$ random oracle) Query.) We first describe the simulation of $\mathrm{H}_{2}$ oracle. On the $i$-th input $\omega_{i}=\left(m_{\ell}, I D_{j}, P K_{k}\right), \mathcal{B}$ first checks if $I D_{j}=I D_{t}$ and $P K_{k}=P K_{t}$. Here $P K_{t}$ is the original public key. We divide into two cases:

1. If $I D_{j}=I D_{t}$ and $P K_{k}=P K_{t}$, then $\mathcal{B}$ flips a biased coin which outputs a value $c_{i}=1$ with probability $\xi$, and $c_{i}=0$ with probability $1-\xi$. The value of $\xi$ will be optimized later.

- If $c_{i}=1, \mathcal{B}$ randomly chooses $h^{\prime}{ }_{i} \in_{R} \mathbb{Z}_{p}^{*}$ where $h^{\prime}{ }_{i} \notin\left\{h_{1}, \ldots, h_{k}\right\}$ and returns $h^{\prime}{ }_{i}$ to $\mathcal{A}$ as the value of $H_{2}\left(\omega_{i}\right)$.

- If $c_{i}=0, \mathcal{B}$ randomly chooses $h^{\prime \prime}{ }_{i} \in_{R}\left\{h_{1}, \ldots, h_{k}\right\}$ as the output of $H_{2}\left(\omega_{i}\right)$, where $h^{\prime \prime}{ }_{i}$ must be a fresh value which has not been assigned as an output of $\mathrm{H}_{2}$ queries before.

2. Otherwise, $\mathcal{B}$ randomly chooses $\mu_{i} \in R \mathbb{Z}_{p}^{*}$ as the output.

In all cases, $\mathcal{B}$ records $\left(\omega_{i}, h^{\prime}{ }_{i}, c_{i}\right),\left(\omega_{i}, h^{\prime \prime}{ }_{i}, c_{i}\right)$ or $\left(\omega_{i}, \mu_{i}\right)$ to another list $L_{H_{2}}$.

For each signing query on an input $\left(m_{\ell}, I D_{j}, P K_{j}\right)$, output $\perp$ if $I D_{j}$ has not been created. Otherwise, we assume that $\mathcal{A}$ has already queried the random oracle $H_{2}$ on the input $\omega_{i}=\left(m_{\ell}, I D_{j}, P K_{j}\right)$. We divide into two cases:

1. If $I D_{j} \neq I D_{t}, \mathcal{B}$ uses the the user secret key and certificate of $I D_{j}$ from the list $L_{k}$ and $\mu_{i}=H_{2}\left(\omega_{i}\right)$ from the list $L_{H_{2}}$ to generate a valid signature.

2. If $I D_{j}=I D_{t}, \mathcal{B}$ first checks the list $L_{H_{2}}$.

- If $c_{i}=1, \mathcal{B}$ reports failure and terminates the simulation.

- Otherwise, that is $c_{i}=0$ and $h^{\prime \prime}{ }_{i}=H_{2}\left(m_{\ell}, I D_{t}, P K_{t}\right)$ is on the list $L_{H_{2}}$. For simplicity, we assume $h^{\prime \prime}{ }_{i}=h_{i} \in\left\{h_{1}, \ldots, h_{k}\right\}$. B outputs $\sigma_{i}=\left(g^{a b}\right)^{\left(x+h_{i}\right)^{-1}}$ as the signature. It is a valid signature because $e\left(\sigma_{i}, P K_{t} \cdot g^{h_{i}}\right)=e\left(\left(g^{a b}\right)^{\left(x+h_{i}\right)^{-1}}, g^{x} \cdot g^{h_{i}}\right)=e\left(g^{a b}, g\right)=e\left(g^{b}, g^{a}\right)=$ $e\left(H_{1}\left(I D_{t}, P K_{t}\right), g_{1}\right)$

Forgery. After all queries, $\mathcal{A}$ outputs $\left(I D^{*}, P K^{*}, m^{*}, \sigma^{*}\right)$. If $\mathcal{A}$ wins, we have the following conditions satisfied:

1. $I D^{*}$ has not been submitted to certification query.

2. $\left(I D^{*}, P K^{*}, m^{*}\right)$ has not been submitted to signing query.

3. $\sigma^{*}$ is a valid signature on $\left(I D^{*}, P K^{*}, m^{*}\right)$. That is,

$$
\begin{aligned}
e\left(\sigma^{*}, P K^{*} \cdot g^{h^{*}}\right) & =e\left(H_{1}\left(I D^{*}, P K^{*}\right), g_{1}\right) \\
e\left(\sigma^{*}, g^{x^{*}} \cdot g^{h^{*}}\right) & =e\left(g^{b}, g^{a}\right) \\
e\left(\sigma^{*}, g\right) & =e\left(\left(g^{a b}\right)^{\left(x^{*}+h^{*}\right)^{-1}}, g\right) \\
\Rightarrow \quad \sigma^{*} & =\left(g^{a b}\right)^{\left(x^{*}+h^{*}\right)^{-1}}
\end{aligned}
$$

where $h^{*}=H_{2}\left(m^{*}, I D^{*}, P K^{*}\right)$ and $P K^{*}=g^{x^{*}}$. 
4. $P K^{*}$ is either the original public key generated by the User-key-gen oracle, or a new public key inputted by $\mathcal{A}$ to the key-replace oracle successfully. Note that in the latter case, $\mathcal{B}$ also knows the corresponding secret key.

If $I D^{*} \neq I D_{t}$, then $\mathcal{B}$ outputs failure and terminates the simulation. Otherwise, $\mathcal{B}$ checks the list $L_{H_{2}}$. If $c^{*}=0, \mathcal{B}$ outputs failure and terminates the simulation. Otherwise, that is, $c^{*}=1$ and $h^{*} \notin\left\{h_{1}, \ldots, h_{k}\right\}$. We divide into two cases:

1. $P K^{*}$ is the original public key generated by user-key-gen oracle. In this case, $\mathcal{B}$ outputs a new pair $\left(h^{*}, \sigma^{*}\right)=\left(h^{*},\left(g^{a b}\right)^{\left(x+h^{*}\right)^{-1}}\right)$ which is the solution of the modified $k$-CAA problem.

2. $P K^{*}$ is a new public key inputted by $\mathcal{A}$ to the key-replace oracle successfully. In this case, $\mathcal{B}$ retrieves the corresponding secret key $x^{*}$ from the list $L_{k} . \mathcal{B}$ computes $\left(\sigma^{*}\right)^{\left(x^{*}+h^{*}\right)}=\left(\left(g^{a b}\right)^{\left(x^{*}+h^{*}\right)^{-1}}\right)^{\left(x^{*}+h^{*}\right)}=g^{a b}$ as the solution of the Weak Modified $k$-CAA problem.

Probability Analysis. $\mathcal{B}$ succeeds if: (1) $E_{1}: \mathcal{B}$ does not abort during simulation; (2) $E_{2}: \mathcal{A}$ wins; and (3) $E_{3}: I D^{*}=I D_{t}$ and $c^{*}=1$.

The advantage of $\mathcal{B}$ is $\epsilon^{\prime}=\operatorname{Pr}\left[E_{1} \wedge E_{2} \wedge E_{3}\right]=\operatorname{Pr}\left[E_{1}\right] \cdot \operatorname{Pr}\left[E_{2} \mid E_{1}\right] \cdot \operatorname{Pr}\left[E_{3} \mid E_{1} \wedge E_{2}\right]$ If $E_{1}$ happens, then:

- $\mathcal{B}$ does not output failure during the simulation of the certification query. This happens with probability $\left(1-\frac{1}{q_{k}}\right)^{q_{e}}$.

- $\mathcal{B}$ does not output failure during the simulation of the corruption query. This happens with probability $\left(1-\frac{1}{q_{k}}\right)^{q_{r}}$.

$-\mathcal{B}$ does not output failure during the simulation of the signing query. This happens with probability $\left(1-\frac{1}{q_{k}} \xi\right)^{q_{s}} \geq(1-\xi)^{q_{s}}$.

Now we have $\operatorname{Pr}\left[E_{1}\right] \geq\left(1-\frac{1}{q_{k}}\right)^{q_{e}+q_{r}}(1-\xi)^{q_{s}}$ In addition, $\operatorname{Pr}\left[E_{2} \mid E_{1}\right]=\epsilon$ and $\operatorname{Pr}\left[E_{3} \mid E_{1} \wedge E_{2}\right]=\frac{\xi}{q_{k}}$ Combine together, we have $\epsilon^{\prime} \geq\left(1-\frac{1}{q_{k}}\right)^{q_{e}+q_{r}}(1-\xi)^{q_{s}} \frac{\xi}{q_{k}} \epsilon$ The function $(1-\xi)^{q_{s}} \xi$ is maximized when $\xi=\frac{1}{q_{s}+1}$. Finally, we have $\epsilon^{\prime} \geq$ $\left(1-\frac{1}{q_{k}}\right)^{q_{e}+q_{r}}\left(1-\frac{1}{q_{s}+1}\right)^{q_{s}} \frac{1}{\left(q_{s}+1\right) q_{k}} \epsilon$ The time $\mathcal{B}$ has used in the simulation is approximately the same as $\mathcal{A}$.

Theorem 2 (Game 2). The CBS scheme proposed in this section is $(\epsilon, t)$ existential unforgeable against Game 2 adversary with advantage at most $\epsilon$ and runs in time at most $t$, assuming that the $\left(\epsilon^{\prime}, t^{\prime}\right) k$-CAA assumption holds in $\mathbb{G}$, where $\epsilon^{\prime} \geq\left(1-\frac{1}{q_{k}}\right)^{q_{r}}\left(1-\frac{1}{q_{s}+1}\right)^{q_{s}} \frac{1}{\left(q_{s}+1\right) q_{k}} \epsilon, t^{\prime}=t$ where $q_{k}, q_{r}, q_{s}$ are the numbers of queries made to the User-key-gen Query, Corruption Query and Signing Query respectively.

Proof. Assume there exists a Game 2 adversary $\mathcal{A}$. We are going to construct another PPT $\mathcal{B}$ that makes use of $\mathcal{A}$ to solve the $k$-CAA problem with probability at least $\epsilon^{\prime}$ and in time at most $t^{\prime}$. We use a similar approach as in 14 .

Let $g$ be a generator of $\mathbb{G}$. Let $x, h_{1}, \ldots, h_{k} \in \mathbb{Z}_{p}^{*}$ be $k+1$ integers. $\mathcal{B}$ is given the problem instance $g, g^{x},\left(h_{1}, g^{\left(x+h_{1}\right)^{-1}}\right), \ldots,\left(h_{k}, g^{\left(x+h_{k}\right)^{-1}}\right) . \mathcal{B}$ is asked to output $\left(h^{*}, g^{\left(x+h^{*}\right)^{-1}}\right)$ for some $h^{*} \notin\left\{h_{1}, \ldots, h_{k}\right\}$. 
Setup. $\mathcal{B}$ simulates the environment and the oracles which $\mathcal{A}$ can access. We

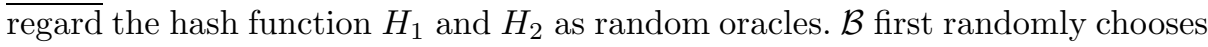
$s \in \in_{R} \mathbb{Z}_{p}^{*}$ and sets $g_{1}=g^{s}$ and gives the parameter param $=\left(e, \mathbb{G}_{1} \mathbb{G}_{T}, p, g, g_{1}\right)$, together with the master secret key $s$ to $\mathcal{A}$.

Oracles Simulation. $\mathcal{B}$ simulates all oracles as follow. Note that different from Game 1, there is no Certification Query, as $\mathcal{A}$ has already obtained the master secret key.

(User-key-gen (and $H_{1}$ random oracle) Query .) At the beginning $\mathcal{B}$ first randomly chooses a number $t \in\left\{1, \ldots, q_{k}\right\}$. $\mathcal{B}$ also maintains a list $L_{U}$ which stores the secret/public key pair of the corresponding identity. $\mathcal{A}$ can query this oracle by submitting an identity $I D_{i}$. If the public key pair is already created, $\mathcal{B}$ retrieves them from the list $L_{U}$. Otherwise,

1. If $i \neq t, \mathcal{B}$ chooses $d_{i}, x_{i} \in R \mathbb{Z}_{p}^{*}$ and sets $P K_{i}=g^{x_{i}}, H_{1}\left(I D_{i}, P K_{i}\right)=g^{d_{i}}$. The corresponding certificate is $C_{I D_{i}}=H_{1}\left(I D_{i}, P K_{i}\right)^{s}=\left(g^{s}\right)^{d_{i}}$ and the user secret key is $x_{i} . \mathcal{B}$ stores $\left(d_{i}, x_{i}, P K_{i}, I D_{i}\right)$ to the list $L_{U}$.

2. If $i=t, \mathcal{B}$ randomly chooses $d_{t} \in_{R} \mathbb{Z}_{p}^{*}$, sets $P K_{i}=g^{x}$ and $H_{1}\left(I D_{i}, P K_{i}\right)=$ $g^{d_{t}}$. In this case, the user secret key is unknown to $\mathcal{B}$.

(Corruption Query.) Given an identity $I D_{i}, \mathcal{B}$ outputs $\perp$ if $I D_{i}=I D_{t}$ or $I D_{i}$ has not been created. Otherwise, $\mathcal{B}$ returns $x_{i}$ from the list $L_{U}$.

(Signing (and $\mathrm{H}_{2}$ random oracle) Query.) We first describe the simulation of $\mathrm{H}_{2}$ random oracle. On the $i$-th input $\omega_{i}=\left(m_{\ell}, I D_{j}, P K_{k}\right), \mathcal{B}$ first checks if $I D_{j}=$ $I D_{t}$ and $P K_{k}=P K_{t}$. We divide into two cases:

1. If $I D_{j}=I D_{t}$ and $P K_{k}=P K_{t}$, then $\mathcal{B}$ flips a biased coin which outputs a value $c_{i}=1$ with probability $\xi$, and $c_{i}=0$ with probability $1-\xi$. The value of $\xi$ will be optimized later.

- If $c_{i}=1, \mathcal{B}$ randomly chooses $h^{\prime}{ }_{i} \in \mathbb{Z}_{R} \mathbb{Z}_{p}$ where $h^{\prime}{ }_{i} \notin\left\{h_{1}, \ldots, h_{k}\right\}$ and returns $h^{\prime}{ }_{i}$ to $\mathcal{A}$ as the value of $H_{2}\left(\omega_{i}\right)$.

- If $c_{i}=0, \mathcal{B}$ randomly chooses $h^{\prime \prime}{ }_{i} \in_{R}\left\{h_{1}, \ldots, h_{k}\right\}$ as the output of $H_{2}\left(\omega_{i}\right)$, where $h^{\prime \prime}{ }_{i}$ must be a fresh value which has not been assigned as an output of $\mathrm{H}_{2}$ queries before.

2. Otherwise, $\mathcal{B}$ randomly chooses $\mu_{i} \in{ }_{R} \mathbb{Z}_{p}^{*}$ as the output.

In all cases, $\mathcal{B}$ records $\left(\omega_{i}, h^{\prime}{ }_{i}, c_{i}\right),\left(\omega_{i}, h^{\prime \prime}{ }_{i}, c_{i}\right)$ or $\left(\omega_{i}, \mu_{i}\right)$ to another list $L_{H_{2}}$.

For each signing query on an input $\left(m_{\ell}, I D_{j}, P K_{j}\right)$, output $\perp$ if $I D_{j}$ has not been created. Otherwise, we assume that $\mathcal{A}$ has already queried the random oracle $H_{2}$ on the input $\omega_{i}=\left(m_{\ell}, I D_{j}, P K_{j}\right)$. We divide into two cases:

1. If $I D_{j} \neq I D_{t}, \mathcal{B}$ uses the the user secret key and certificate of $I D_{j}$ from the list $L_{k}$ and $\mu_{i}=H_{2}\left(\omega_{i}\right)$ from the list $L_{H_{2}}$ to generate a valid signature.

2. If $I D_{j}=I D_{t}, \mathcal{B}$ first checks the list $L_{H_{2}}$.

- If $c_{i}=1, \mathcal{B}$ reports failure and terminates the simulation.

- Otherwise, that is $c_{i}=0$ and $h^{\prime \prime}{ }_{i}=H_{2}\left(m_{\ell}, I D_{t}, P K_{t}\right)$ is on the list $L_{H_{2}}$. For simplicity, we assume $h^{\prime \prime}{ }_{i}=h_{i} \in\left\{h_{1}, \ldots, h_{k}\right\}$. B outputs 


$$
\begin{aligned}
& \sigma_{i}=\left(g^{s d_{t}}\right)^{\left(x+h_{i}\right)^{-1}} \text { as the signature. It is a valid signature because } \\
& e\left(\sigma_{i}, P K_{t} \cdot g^{h_{i}}\right)=e\left(\left(g^{s d_{t}}\right)^{\left(x+h_{i}\right)^{-1}}, g^{x} \cdot g^{h_{i}}\right)=e\left(g^{s d_{t}}, g\right)=e\left(g^{d_{t}}, g^{s}\right) \\
& =e\left(H_{1}\left(I D_{t}, P K_{t}\right), g_{1}\right)
\end{aligned}
$$

Forgery. After all queries, $\mathcal{A}$ outputs $\left(I D^{*}, P K^{*}, m^{*}, \sigma^{*}\right)$. If $\mathcal{A}$ wins, we have the following conditions satisfied:

1. $P K^{*}$ has not been submitted to corruption query.

2. $\left(I D^{*}, P K^{*}, m^{*}\right)$ has not been submitted to signing query.

3. $\sigma^{*}$ is a valid signature on $\left(I D^{*}, P K^{*}, m^{*}\right)$. That is,

$$
\begin{aligned}
e\left(\sigma^{*}, P K^{*} \cdot g^{h^{*}}\right) & =e\left(H_{1}\left(I D^{*}, P K^{*}\right), g_{1}\right) \\
e\left(\sigma^{*}, g^{x} \cdot g^{h^{*}}\right) & =e\left(g^{d_{t}}, g^{s}\right) \\
e\left(\sigma^{*}, g\right) & =e\left(\left(g^{s d_{t}}\right)^{\left(x+h^{*}\right)^{-1}}, g\right) \\
\Rightarrow \quad \sigma^{*} & =\left(g^{s d_{t}}\right)^{\left(x+h^{*}\right)^{-1}}
\end{aligned}
$$

where $h^{*}=H_{2}\left(m^{*}, I D^{*}, P K^{*}\right)$ and $P K^{*}=g^{x^{*}}$.

If $I D^{*} \neq I D_{t}$, then $\mathcal{B}$ outputs failure and terminates the simulation. Otherwise, $\mathcal{B}$ checks the list $L_{H_{2}}$. If $c^{*}=0, \mathcal{B}$ outputs failure and terminates the simulation. Otherwise, that is, $c^{*}=1$ and $h^{*} \notin\left\{h_{1}, \ldots, h_{k}\right\} \cdot \mathcal{B}$ computes $\phi=\left(\sigma^{*}\right)^{\left(s d_{t}\right)^{-1}}=$ $g^{\left(x+h^{*}\right)^{-1}}$ and outputs $\left(h^{*}, \phi\right)$ as the solution of the problem instance.

Probability Analysis. $\mathcal{B}$ succeeds if: (1) $E_{1}: \mathcal{B}$ does not abort during simulation; (2) $E_{2}: \mathcal{A}$ wins; and (3) $E_{3}: I D^{*}=I D_{t}$ and $c^{*}=1$.

The advantage of $\mathcal{B}$ is $\epsilon^{\prime}=\operatorname{Pr}\left[E_{1} \wedge E_{2} \wedge E_{3}\right]=\operatorname{Pr}\left[E_{1}\right] \cdot \operatorname{Pr}\left[E_{2} \mid E_{1}\right] \cdot \operatorname{Pr}\left[E_{3} \mid E_{1} \wedge E_{2}\right]$ If $E_{1}$ happens, then:

- $\mathcal{B}$ does not output failure during the simulation of the corruption query. This happens with probability $\left(1-\frac{1}{q_{k}}\right)^{q_{r}}$.

$-\mathcal{B}$ does not output failure during the simulation of the signing query. This happens with probability $\left(1-\frac{1}{q_{k}} \xi\right)^{q_{s}} \geq(1-\xi)^{q_{s}}$.

Now we have $\operatorname{Pr}\left[E_{1}\right] \geq\left(1-\frac{1}{q_{k}}\right)^{q_{r}}(1-\xi)^{q_{s}}$ In addition, $\operatorname{Pr}\left[E_{2} \mid E_{1}\right]=\epsilon$ and $\operatorname{Pr}\left[E_{3} \mid E_{1} \wedge E_{2}\right]=\frac{\xi}{q_{k}}$ Combine together, we have $\epsilon^{\prime} \geq\left(1-\frac{1}{q_{k}}\right)^{q_{r}}(1-\xi)^{q_{s}} \frac{\xi}{q_{k}} \epsilon$ The function $(1-\xi)^{q_{s}} \xi$ is maximized when $\xi=\frac{1}{q_{s}+1}$. Finally, we have $\epsilon^{\prime} \geq(1-$ $\left.\frac{1}{q_{k}}\right)^{q_{r}}\left(1-\frac{1}{q_{s}+1}\right)^{q_{s}} \frac{1}{\left(q_{s}+1\right) q_{k}} \epsilon$ The time $\mathcal{B}$ has used in the simulation is approximately the same as $\mathcal{A}$.

\section{Conclusion}

We have proposed a short CBS scheme. It is the shortest CBS scheme in the literature. The signature size and public key are just one group element respectively. It is even shorter than any PKI based signature, which require at least one group element for the signature size, public key and certificate respectively. Our scheme can be proven secure in the random oracle model using the weak 
modified $k$-CAA assumption. The computation requirement is very light, can be implemented in many devices easily. We believe our scheme can be very useful in many different applications such as wireless sensor networks and mesh networks, wherever space efficiency and level of trust are the major concerns.

\section{References}

1. Al-Riyami, S.S., Paterson, K.: Certificateless public key cryptography. In: Laih, C.-S. (ed.) ASIACRYPT 2003. LNCS, vol. 2894, pp. 452-473. Springer, Heidelberg (2003)

2. Au, M., Chen, J., Liu, J., Mu, Y., Wong, D., Yang, G.: Malicious KGC attacks in certificateless cryptography. In: ASIACCS 2007, pp. 302-311. ACM Press, New York (2007), http://eprint.iacr.org/2006/255

3. Au, M.H., Liu, J.K., Susilo, W., Yuen, T.H.: Certificate based (Linkable) ring signature. In: Dawson, E., Wong, D.S. (eds.) ISPEC 2007. LNCS, vol. 4464, pp. 79-92. Springer, Heidelberg (2007)

4. Boneh, D., Franklin, M.: Identity-Based Encryption from the Weil Pairing. In: Kilian, J. (ed.) CRYPTO 2001. LNCS, vol. 2139, pp. 213-229. Springer, Heidelberg (2001)

5. Boneh, D., Lynn, B., Shacham, H.: Short signatures from the weil pairing. In: Boyd, C. (ed.) ASIACRYPT 2001. LNCS, vol. 2248, pp. 514-532. Springer, Heidelberg (2001)

6. Gentry, C.: Certificate-based encryption and the certificate revocation problem. In: Biham, E. (ed.) EUROCRYPT 2003. LNCS, vol. 2656, pp. 272-293. Springer, Heidelberg (2003)

7. Girault, M.: Self-certified public keys. In: Davies, D.W. (ed.) EUROCRYPT 1991. LNCS, vol. 547, pp. 490-497. Springer, Heidelberg (1991)

8. Hess, F.: Efficient identity based signature schemes based on pairings. In: Nyberg, K., Heys, H.M. (eds.) SAC 2002. LNCS, vol. 2595, pp. 310-324. Springer, Heidelberg (2003)

9. Kang, B.G., Park, J.H., Hahn, S.G.: A certificate-based signature scheme. In: Okamoto, T. (ed.) CT-RSA 2004. LNCS, vol. 2964, pp. 99-111. Springer, Heidelberg (2004)

10. Li, J., Huang, X., Mu, Y., Susilo, W., Wu, Q.: Certificate-based signature: Security model and efficient construction. In: López, J., Samarati, P., Ferrer, J.L. (eds.) EuroPKI 2007. LNCS, vol. 4582, pp. 110-125. Springer, Heidelberg (2007)

11. Liu, J.K., Baek, J., Susilo, W., Zhou, J.: Certificate-based signature schemes without pairings or random oracles. In: Wu, T.-C., Lei, C.-L., Rijmen, V., Lee, D.-T. (eds.) ISC 2008. LNCS, vol. 5222, pp. 285-297. Springer, Heidelberg (2008)

12. Mitsunari, S., Sakai, R., Kasahara, M.: A new traitor tracing. IEICE Transactions E85-A(2), 481-484 (2002)

13. Shamir, A., Tauman, Y.: Improved online/Offline signature schemes. In: Kilian, J. (ed.) CRYPTO 2001. LNCS, vol. 2139, pp. 355-367. Springer, Heidelberg (2001)

14. Tso, R., Yi, X., Huang, X.: Efficient and short certificateless signature. In: Franklin, M.K., Hui, L.C.K., Wong, D.S. (eds.) CANS 2008. LNCS, vol. 5339, pp. 64-79. Springer, Heidelberg (2008) 